\title{
Proprotein convertase subtilisin/kexin type 9 expression is transiently up-regulated in the acute period of myocardial infarction in rat
}

Yan Zhang ${ }^{\dagger}$, Jun Liư ${ }^{\dagger}$, Sha Li, Rui-Xia Xu, Jing Sun, Yue Tang ${ }^{*}$ and Jian-Jun Li

\begin{abstract}
Background: The proprotein convertase subtilisin/kexin type 9 (PCSK9) has been confirmed as a major factor regulating cholesterol homeostasis and has low-density lipoprotein receptor (LDLR) independent effects. In addition, the pathogenesis of acute myocardial infarction (AMI) involves lipids alteration and other acute phase responses. It remains unknown whether the PCSK9 expression is influenced by the impact of AMI. The present study aimed to investigate the changes of PCSK9 concentration using AMI rat model.

Methods: AMI ( $n=6-8$ at each time point) or sham operated $(n=6)$ adult male rats model were used. Whole blood and liver tissue were collected at 1, 3, 6, 9, 12, 24, 48, and 96 hour (h) post infarction. The plasma PCSK9 concentration was measured by ELISA and lipid profiles were measured by enzymatic assay. The liver mRNA levels of PCSK9, LDLR, sterol response element binding protein-2 (SREBP-2) and hepatocyte nuclear factor 1a (HNF1a) were measured by quantitative real-time PCR.

Results: The plasma PCSK9 concentration was increased from $12 \mathrm{~h}$ to $96 \mathrm{~h}(\mathrm{P}<0.05 \mathrm{vs}$. control). Paralleled with the enhanced plasma PCSK9 concentration, the hepatic PCSK9 mRNA expression was up-regulated by 2.2 -fold at $12 \mathrm{~h}$ and 4.1 -fold at $24 \mathrm{~h}$. Hepatic mRNA levels of LDLR, SREBP-2 and HNF1 $a$ were all increased and lipid profiles underwent great changes at this acute period.

Conclusions: We firstly demonstrated that PCSK9 was transiently up-regulated in the acute period of AMl, which is also driven by transcriptional factors, SREBP-2 and HNF1a, suggesting that the role of PCSK9 in myocardial injury may be needed further study.
\end{abstract}

Keywords: Acute myocardial infarction, PCSK9, Rat

\section{Background}

Proprotein convertase subtilisin/kexin type 9 (PCSK9), originally discovered as a third gene involved in autosomal dominant hypercholesterolemia [1], has gained considerable attention over the past decade. A number of studies have demonstrated that, a high level of plasma PCSK9, caused by gain-of-function mutations in human or over-expression in animal model, exclusively resulted in increased serum concentration of low-density lipoproteincholesterol (LDL-C), and that the patients with gain-of-

\footnotetext{
*Correspondence: tangyue1226@vip.sina.com; lijianjun938@126.com ${ }^{\dagger}$ Equal contributors

Division of Dyslipidemia, State Key Laboratory of Cardiovascular Disease, FuWai Hospital, National Center for Cardiovascular Diseases, Chinese

Academy of Medical Sciences, Peking Union Medical College, BeiLiShi Road 167, Beijing 100037, China
}

function mutations presented with increased cardiovascular risk [2-5]. Conversely, loss-of-function mutations in PCSK9 and knockdown of PCSK9 gene caused not only a low plasma level of PCSK9, but also a significant decreased level of LDL-C, accompanied by decreased cardiovascular risk [5,6]. Recent studies have elucidated that PCSK9 is mainly synthesized by the liver and has been shown to bind to the low-density lipoprotein receptor (LDLR), subsequently promoting its degradation [7]. This process reduces the availability of LDLR, the major receptor mediating the clearance of low-density lipoprotein (LDL) particle, and results in increased plasma LDL-C levels. Since elevated LDL-C has long been established as a predominant risk factor for coronary artery disease (CAD), manipulating PCSK9 level would be a promising new treatment strategy [8]. 
The pathogenesis of acute myocardial infarction (AMI) is multifactorial, however, several studies have indicated that hyperlipidemia is a major risk factor accountable for $54 \%$ of population-attributable risk for AMI [9]. In addition, previous studies have reported that during the acute stage of AMI, serum lipids metabolism was severely affected [10]. Whereas PCSK9, an important factor regulating cholesterol homeostasis, has been reported to be associated with a variety of physiological and pathological factors dependent or independent of the LDLR, such as statins [11], fenofibrate [12], fasting [13], sex [14], periodontal infection [15], systemic inflammation [16], severe trauma injury [17] and the severity of coronary atherosclerosis [18]. However, it remains unknown whether the PCSK9 expression is influenced by the impact of AMI. Therefore, the aim of present study was to investigate the changes of both plasma and liver PCSK9 level using the AMI rat model.

\section{Methods}

\section{Animal model}

Adult male Sprague-Dawley rats $(n=6-8$ at each time point), weighing 260-280 g, were acclimatized with a 12 hour (h) light/dark cycle at a controlled room temperature of $22-24^{\circ} \mathrm{C}$, and rats were allowed free access to the regular and normal diet (fat content 4.62\%) and clean drinking water for seven days before use. Myocardial infarction models were induced by ligation of the left anterior descending coronary artery (LAD) under ether anesthesia as previously described [19]. Electrocardiography was used to demonstrate ST elevation and thereby confirm the success of surgery. Sham operated animals $(n=6)$ underwent the same procedure except that no ligation was carried out. The experimental procedures were approved by the Institutional Animal Care and Use Committee of the FuWai Hospital and conformed to Guide for the Care and Use of Laboratory Animals from National Institutes of Health.

\section{Blood and tissue sampling}

Prior to sacrifice, $2 \mathrm{~mL}$ fasting blood samples were collected from the tail vein at 1, 3, 6, 9, 12, 24, 48, and $96 \mathrm{~h}$ post infarction and transferred to K2 EDTA tubes. The blood samples were centrifuged, and the plasma was stored at $-80^{\circ} \mathrm{C}$ until the analyses were performed. Immediately after blood sampling, the animals were sacrificed by an overdose of pentobarbital sodium. Tissue fragments from liver were collected and snap-frozen in liquid nitrogen and stored at $-80^{\circ} \mathrm{C}$.

\section{Blood sample measurements}

Concentrations of serum total cholesterol (TC), triglycerides (TG), high-density lipoprotein-cholesterol (HDL-C), LDL-C, and free fatty acid (FFA) were determined on an automatic biochemistry analyzer (Hitachi 7150, Tokyo,
Japan). Plasma PCSK9 concentration was measured using a high-sensitivity, quantitative sandwich enzyme immunoassay (Quantikine ELISA, R\&D Systems Europe Ltd) according to our previous studies [20]. The lower limit of detection was $0.096 \mathrm{ng} / \mathrm{ml}$. The white blood cell counts (WBCC) were determined using an automated blood cell counter (Beckman Coulter Ireland Inc Mervue, Galway, Ireland).

\section{RNA isolation, CDNA synthesis and quantitative real-time PCR}

Following the manufacturer's instructions, the total RNA of liver tissue was isolated using the Trizol reagent Kit (Invitrogen, USA) with the aid of an Omni Tissue Homogenizer, and then was measured by spectrophotometry at an absorbance of $260 \mathrm{~nm}$, and designated the purity valid if the ratio of A260/A280 was in the range of 1.8 to 2.0. The integrity of the RNA was checked by denaturing agarose gel electrophoresis and ethidium bromide staining. First-strand cDNA was synthesized from $2 \mu \mathrm{g}$ of total RNA using revert Aid First Strand cDNA synthesis kit (Promega, USA). Sequences of primers were given in Table 1. The abundances of key genes (PCSK9, LDLR, sterol regulator element binding protein-2 (SREBP-2), hepatocyte nuclear factor $1 \alpha(\mathrm{HNF} 1 \alpha)$ ) and glyceraldehyde-3-phosphate dehydrogenase (GAPDH) mRNA were analyzed by real time quantitative PCR (RT-PCR). RT-PCR reaction was carried out with SYBR Premix ExTaq (TaKaRa Bio Inc. Japan) on 7500HT RT-PCR system (Applied Biosystems, Foster, CA, USA). Standard curves for each primer pair were generated by serial dilutions of cDNA from a reference sample and used for regression analyses. All PCR assays were performed in triplicate. The variance of the triplicate measurements was $<1 \%$. Results were analyzed using the standard curve method by the sequence detection systems (SDS) software. All results were normalized against GAPDH.

\section{Statistical analysis}

Continuous variables were expressed as mean \pm standard deviation (SD). One-way analysis of variance (ANOVA) followed by the Dunnett's T3 test (unequal variances) or Bonferroni test (equal variances) were performed to test

Table 1 Sequence of primers used in real-time RT-PCR

\begin{tabular}{lll}
\hline Gene & Forward primer $\left(5^{\prime} \rightarrow 3^{\prime}\right)$ & Reverse Primer $\left(5^{\prime} \rightarrow 3^{\prime}\right)$ \\
\hline PCSK9 & CGGGAAGGACATCATCGG & GGTTCAGCATCATAGCCACAAT \\
LDLR & GATTGGCTATGAGTGCCTATGTC & GTGAAGAGCAGAAACCCTATGG \\
SREBP2 & AGCATACCGCAAGGTGTTCC & CCAGGTGTCTACTTCTCCGTGT \\
HNF1a & ATGACACGGATGACGATGGG & ATGGGTCCTCCTGAAGAAGTGA \\
GAPDH & ACAGCAACAGGGTGGTGAC & TाTGAGGGTGCAGCGAACTT
\end{tabular}

PCSK9 = proprotein convertases subtilisin kexin 9; LDLR = low-density lipoprotein receptor; SREBP-2 = sterol response element binding protein-2; HNF1 $a$ = hepatocytenuclear factor 1a; GAPDH = glyceraldehyde-3-phosphate dehydrogenase. 
the differences among sham and AMI groups. A P value of less than 0.05 was considered statistical significance. The SPSS 19.0 statistical software package (SPSS Inc., Chicago, IL, USA) was used for all of the statistical analysis.

\section{Results}

Plasma concentration of PCSK9 and WBCC

The plasma concentration of PCSK9 in sham and AMI groups were indicated in Figure 1. Compared with sham group, PCSK9 concentration was significantly increased at $12 \mathrm{~h}(544.18 \pm 262.69 \mathrm{ng} / \mathrm{ml}$ vs. $293.53 \pm 119.90 \mathrm{ng} / \mathrm{ml}$, $\mathrm{P}<0.05$ vs. control) post AMI, and reached a peak level of $1647.29 \pm 120.47 \mathrm{ng} / \mathrm{ml}$ ( $\mathrm{P}<0.05 \mathrm{vs}$. control) at $48 \mathrm{~h}$, then decreased thereafter to reach a value of $467.54 \pm$ $155.73 \mathrm{ng} / \mathrm{ml}(\mathrm{P}<0.05$ vs. control) at $96 \mathrm{~h}$. Meanwhile, we observed that the plasma WBCC level was increased at $3 \mathrm{~h}$ after infarction $\left(6.32 \times 10^{9} / \mathrm{L}\right.$ vs. $4.24 \pm 1.32 \times 10^{9} / \mathrm{L}$ in sham group, $\mathrm{P}<0.05$ vs. control), and continued to rise at $6 \mathrm{~h}$ and $12 \mathrm{~h}\left(9.58 \pm 2.26 \times 10^{9} / \mathrm{L}, 12.74 \pm 3.88 \times 10^{9} / \mathrm{L}\right.$, respectively; both $\mathrm{P}<0.05$ vs. control).

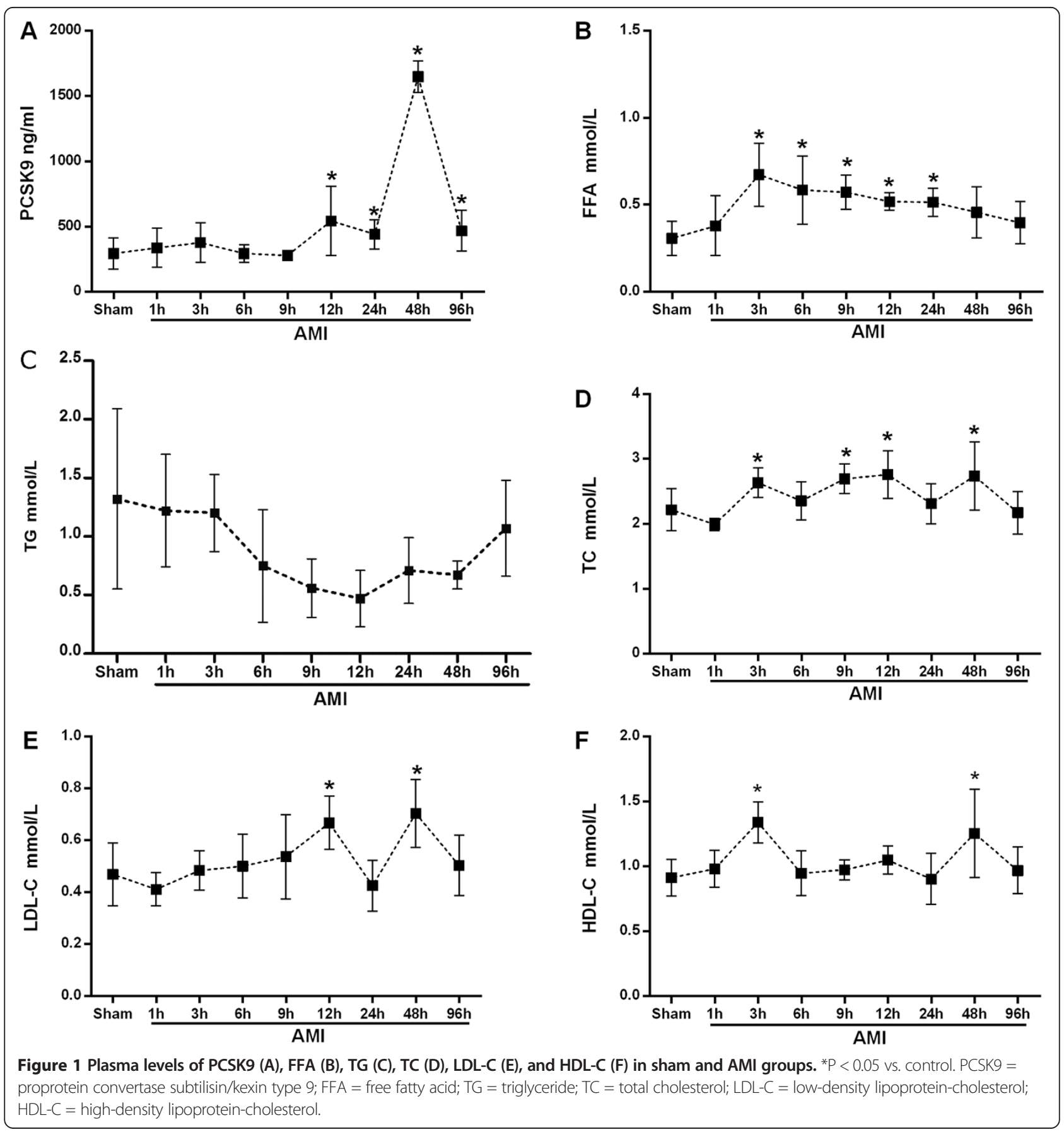




\section{Plasma levels of lipid parameters}

FFA level started to increase at $3 \mathrm{~h}$ after AMI, and maintained a high level until $24 \mathrm{~h}$, then decreased to a level similar to that of the sham group. There was no significant change in TG level from $1 \mathrm{~h}$ to $96 \mathrm{~h}$ even though it showed a decrease and then regressive trend. The TC level exhibited an increase at $3 \mathrm{~h}$ after AMI, and thereafter maintained a high level with intermediate fluctuations at $6 \mathrm{~h}$ and $24 \mathrm{~h}$. Its concentration returned to the sham level at $96 \mathrm{~h}$. There was no significant change in LDL-C level from $1 \mathrm{~h}$ to $96 \mathrm{~h}$ except for two transient increase of LDL-C at $12 \mathrm{~h}$ and $48 \mathrm{~h}$. HDL-C level was steady at most of the time point except for two transient increase at $3 \mathrm{~h}$ and $48 \mathrm{~h}$.

\section{Expression of PCSK9 and LDLR mRNA in AMI rat hepatic cells}

To determine the relative mRNA expression of PCSK9 and LDLR in the liver at different time post AMI, RTPCR was performed. As shown in Figure 2A-B, at $12 \mathrm{~h}$ and $24 \mathrm{~h}$, the amount of PCSK9 mRNA was increased by 2.2 -fold and 4.1 -fold, respectively ( $\mathrm{P}<0.05$ vs. control), then followed by a precipitous drop to a level similar to that of the sham group at $48 \mathrm{~h}$ and $96 \mathrm{~h}$. Expression of the LDLR mRNA, which is known to be associated with PCSK9, was also increased by 4.7 -fold at $12 \mathrm{~h}$, and reached a peak level at $24 \mathrm{~h}$ (increased by 6.7 -fold), but slightly decreased to 4.4 -fold at $48 \mathrm{~h}(\mathrm{P}<0.05$ vs. control). At $96 \mathrm{~h}$, there existed no significant difference compared to the sham group.

\section{Expression of SREBP-2 and HNF1a mRNA in AMI rat hepatic cells}

To determine the transcriptional level mechanism underlying the mRNA alteration of PCSK9 and LDLR at different period of AMI, we quantified SREBP-2 and HNF1 $\alpha$ expression using RT-PCR. The mRNA expression of SREBP-2 (Figure 2C) and HNF1 $\alpha$ (Figure 2D) were both up-regulated at $12 \mathrm{~h}$ (1.9-fold and 6.5-fold, respectively, $\mathrm{P}<0.05$ vs. control) and reached their peak level at $24 \mathrm{~h}$ (2.8-fold and 6.9-fold, respectively, $\mathrm{P}<0.05$ vs. control), then dropped to the level similar to the sham group since $48 \mathrm{~h}$.

\section{Discussion}

PCSK9 has been confirmed as a major factor regulating hepatic LDLR proteins and consequently serum cholesterol levels [7]. Recently, several studies have emphasized that PCSK9 was also involved in the physiology of non-hepatic cells or have LDLR-independent effects $[15,16]$. Interestingly, in the present study our data for the first time found that the plasma PCSK9 concentration was drastically induced from $12 \mathrm{~h}$ to $96 \mathrm{~h}$ at the acute period of AMI in rat model. Paralleled with the enhanced plasma PCSK9 concentration, hepatic PCSK9 mRNA expression was also up-regulated by 2.2 -fold at
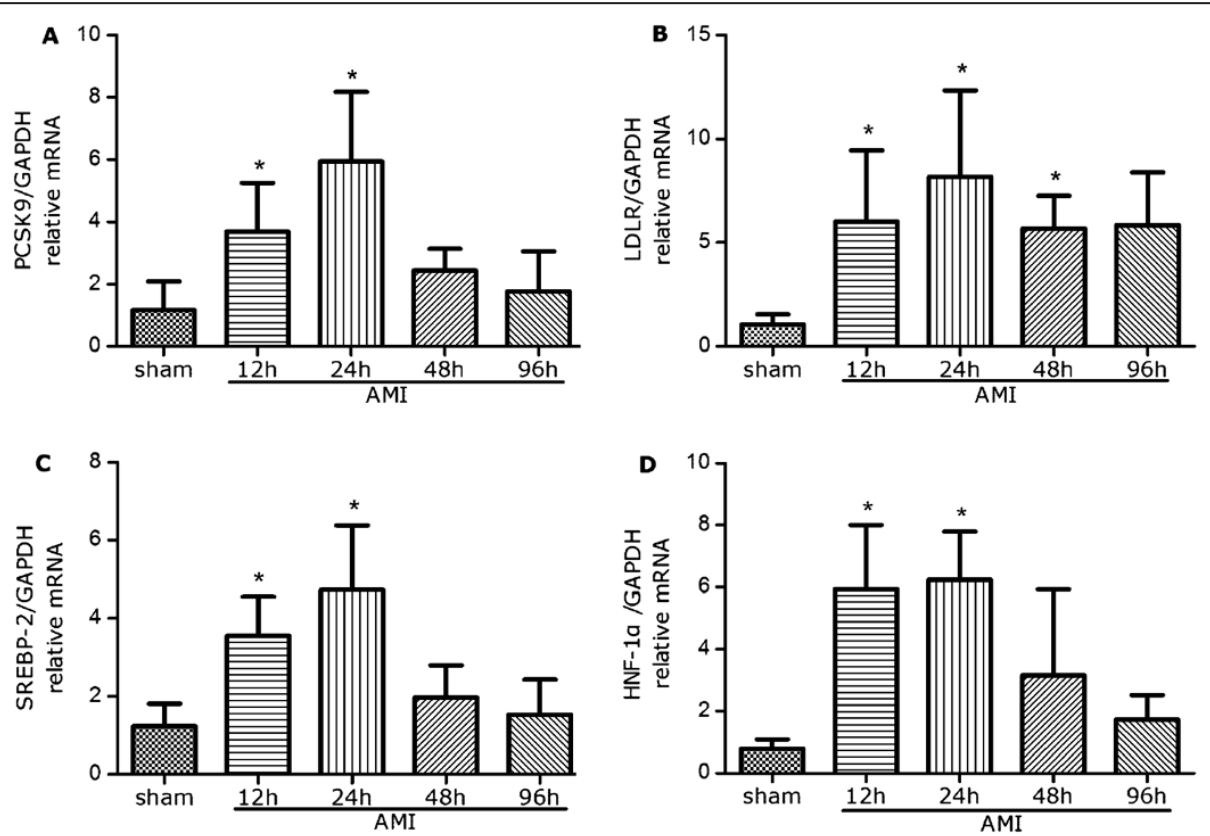

Figure 2 The mRNA expression of PCSK9 (A), LDLR (B), SREBP-2 (C) and HNF1a (D) in the liver were measured using real-time PCR. The relative ratio and standard deviation were calculated using comparative $C T$ method $(\Delta \Delta C T$ value). All results were normalized against GAPDH. ${ }^{*}$ P $<0.05$ vs. control. PCSK9 = proprotein convertase subtilisin/kexin type 9; LDLR =low-density lipoprotein receptor; SREBP-2 $=$ sterol regulator element-binding protein-2; HNF1a= hepatocyte nuclear factor 1a; GAPDH = glyceraldehyde 3-phosphate dehydrogenase. 
$12 \mathrm{~h}$ and 4.1 -fold at $24 \mathrm{~h}$. In consistent with previous studies, the up-regulated PCSK9 expression could be driven by SREBP-2 [21] and HNF1 $\alpha$ [22], both of which are pivotal transcription factors for PCSK9.

The mechanisms accounting for the transiently upregulated PCSK9 level at the acute period of AMI remain unclear. We speculated that it may be correlated with the following factors. Primarily, systemic inflammation may be one of the involving mechanisms. Inflammation is a hallmark throughout the distinct stages of atherosclerotic lesion formation preceding AMI as well as the time of plaque rupture [23]. Several large, prospective epidemiological, clinical and experimental studies converge on inflammation as a pivotal factor in AMI progression and exacerbation $[24,25]$. In the study conducted by Yavuz MT et al. demonstrated that patients with significant coronary stenosis had elevated interleukin-6 (IL-6), high-sensitivity C-reactive protein (hs-CRP) and higher WBCC levels [26]. In the current study, we also observed that the plasma level of WBCC was dramatically increased at the early stage of AMI. Our previous clinical studies $[27,28]$ showed that a persistent and enhanced inflammatory responsiveness to pro-inflammatory stimulators, such as C-reactive protein, are involved in the pathogenesis of acute coronary syndrome. In a study neutrophil to lymphocyte ratio (NLR) was found to have a significant relationship with the severity of ACS assessed by SYNTAX Score and higher NLR was found in ST elevation myocardial infarction patients [29]. Activated neutrophils release a variety of proteolytic enzymes such as myeloperoxidase, which are responsible for tissue injury [30]. Therefore, it has been well established that the pathogenesis of AMI is accompanied with systemic inflammation. Additionally, the study conducted by Feingold KR et al. [16] demonstrated that in mice systemic inflammation induced by lipopolysaccharide or other treatments all resulted in a marked increase in hepatic PCSK9 mRNA levels (4 h-2.5 fold increase; 38 h-12.5 fold increase). Thus, it seems reasonable to consider inflammation as one of the underlying mechanisms. The second mechanism may be associated with the injury induced by AMI. Le Bras M et al. [17] revealed that plasma PCSK9 level was increased by 2 -fold between days 0 and 8 in patients with severe multiple trauma, which may provide evidence that injury may be involved in the up-regulation of PCSK9 during the acute phase of AMI. Finally, necrosis and apoptosis [31] in the process of AMI may be the additional mechanism for the increased PCSK9 concentration. Similar to our study, Rousselet $\mathrm{E}$ et al. demonstrated that ischemic stroke triggered an up-regulation of PCSK9 level [32]. Additionally, $\mathrm{Wu} \mathrm{CY}$ et al. using human endothelial cells demonstrated that oxidized LDL-induced apoptosis resulted elevated expression of PCSK9 and the apoptosis could be inhibited by PCSK9 siRNA [33]. It is plausible to consider that the above factors may contribute to the up-regulated PCSK9 level. However, detailed studies are warranted to explore the exact mechanisms involved in this specific pathological condition.

Although clinical implications of the elevated plasma PCSK9 concentration in the acute period of AMI are still vague, there may be several clinical significances. Firstly, PCSK9 may be an acute-phase response protein (APRP). In our study, we found that plasma PCSK9 concentration was remarkably induced from $12 \mathrm{~h}$ to $96 \mathrm{~h}$ post AMI. The impact of the increased PCSK9 concentration may be correlated to elevated serum LDL-C levels, which could have beneficial effects on host defense [34]. The changing pattern and relating impact of PCSK9 may be in accordance with standard APRP. However, Le Bras $M$ et al. [17] showed that in patients with severe multiple trauma were lack of a rapid increase of circulating PCSK9 level, and posed a challenge to the hypothesis that PCSK9 is an APRP. Thereby, more investigations are required to verify this hypothesis. Secondly, PCSK9 may be a biomarker for future events of AMI. Previous study had demonstrated that at day 8, plasma PCSK9 concentrations were positively associated with clinical severity and acted as a late biomarker of severity illness in the severe trauma patients hospitalized in the intensive care unit [17]. Besides that, PCSK9 has been demonstrated to be expressed in human atherosclerotic plaques [35] and the study investigated the relationship between PCSK9 genotypes/ haplotypes and severity of coronary atherosclerosis elucidated a modest association between minimum lumen diameter tertiles and haplotype 3 [18]. Based on these findings, we tentatively speculated that plasma PCSK9 concentration may be related to the infarct size and severity, which were predicting factors for cardiac remodeling [36] and even cardiac dysfunction. As a result, we suspected that PCSK9 may be a biomarker for the prognosis of AMI. Nevertheless, such hypothesis is being of under investigation in our group. Thirdly, the intensive statin therapy is an essential strategy after AMI [37]. Early administrated with statin can improve the outcome in those patients [38]. Considering the up-regulated PCSK9 expression by statin and AMI, adding PCSK9 inhibitors may bring greater benefits for those patients. Such hypothesis is also needed to be confirmed in the future.

Our study has several limitations. First, we did not observe the long-term impact of the enhanced PCSK9 expression on the prognosis parameters in the AMI rat model. Second, we did not evaluate the protein levels of PCSK9 and LDLR, which may provide more evidence about the relationship between PCSK9 and LDLR. 


\section{Conclusions}

In summary, we demonstrated for the first time that plasma PCSK9 concentration was significantly induced at $12 \mathrm{~h}$ after AMI, and still higher than the sham group at $96 \mathrm{~h}$, and this result was further confirmed by the increased liver mRNA levels. At the transcriptional level, SREBP-2 and HNF1 $\alpha$ were the predominate factors that activated the expression of PCSK9. The role of PCSK9 in myocardial injury may be needed further study.

\section{Abbreviations}

PCSK9: Proprotein convertase subtilisin/kexin type 9; LDL-C: Low-density lipoprotein-cholesterol; LDLR: Low-density lipoprotein receptor; SREBP-2: Sterol regulator element-binding protein-2; AMl: Acute myocardial infarction.

\section{Competing interests}

The authors declare that they have no competing interests.

\section{Authors' contributions}

$Z Y$ and $\sqcup$ completed the project, analyzed the data, and wrote the manuscript. L-J and TY established the study, interpreted the data, and contributed to reviewing/editing the manuscript. LS, XR-X, and SJ contributed to assay and analyzing the data. All authors read and approved the final manuscript.

\section{Acknowledgments}

This work was partly supported by National Natural Scientific Foundation (81070171, 81241121), Specialized Research Fund for the Doctoral Program of Higher Education of China (20111106110013), Capital Special Foundation of Clinical Application Research (Z121107001012015), Capital Health Development Fund (2011400302), and Beijing Natural Science Foundation (7131014) awarded to Dr. Jian-Jun Li, MD, PhD.

Received: 12 August 2014 Accepted: 11 December 2014 Published: 17 December 2014

\section{References}

1. Cariou B, May CL, Costet P: Clinical aspects of PCSK9. Atherosclerosis 2011, 216:258-265.

2. Homer VM, Marais AD, Charlton F, Laurie AD, Hurndell N, Scott R, Mangili F, Sullivan DR, Barter PJ, Rye KA, George PM, Lambert G: Identification and characterization of two non-secreted PCSK9 mutants associated with familial hypercholesterolemia in cohorts from New Zealand and South Africa. Atherosclerosis 2008, 196:659-666.

3. Wu NQ Guo YL, Xu RX, Liu J, Zhu CG, Jiang LX, Li JJ: Acute myocardial infarction in an 8-year old male child with homozygous familiar hypercholesterolemia: laboratory findings and response to lipidlowering drugs. Clin Lab 2013, 59:901-907.

4. Benn M, Watts GF, Tybjaerg-Hansen A, Nordestgaard BG: Familial hypercholesterolemia in the danish general population: prevalence, coronary artery disease, and cholesterol-lowering medication. J Clin Endocrinol Metab 2012, 97:3956-3964

5. Denis M, Marcinkiewicz J, Zaid A, Gauthier D, Poirier S, Lazure C, Seidah NG, Prat A: Gene Inactivation of proprotein convertase subtilisin/kexin type 9 reduces atherosclerosis in mice. Circulation 2012, 125:894-901.

6. Cohen JC, Boerwinkle E, Mosley TH Jr, Hobbs HH: Sequence variations in PCSK9, low LDL, and protection against coronary heart disease. N Engl J Med 2006, 354:1264-1272

7. Lagace TA, Curtis DE, Garuti R, McNutt MC, Park SW, Prather HB, Anderson NN, Ho YK, Hammer RE, Horton JD: Secreted PCSK9 decreases the number of LDL receptors in hepatocytes and in livers of parabiotic mice. J Clin Invest 2006, 116:2995-3005

8. Urban D, Pöss J, Böhm M, Laufs U: Targeting the Proprotein Convertase Subtilisin/Kexin Type 9 for the Treatment of Dyslipidemia and Atherosclerosis. J Am Coll Cardiol 2013, 62:1401-1408.

9. Yusuf S, Hawken S, Ounpuu S, Dans T, Avezum A, Lanas F, McQueen M, Budaj A, Pais P, Varigos J, Lisheng L, INTERHEART Study Investigators: Effect of potentially modifiable risk factors associated with myocardial infarction in 52 countries (the INTERHEART study): case-control study. Lancet 2004, 364:937-952
10. Balci B: The modification of serum lipids after acute coronary syndrome and importance in clinical practice. Curr Cardiol Rev 2011, 7:272-276.

11. Liu J, Guo YL, Xu RX, Li JJ: Rapid effects of different lipid-lowering drugs on PCSK9 in humans. Clin Lipidol 2013, 8:519-524.

12. Troutt JS, Alborn WE, Cao G, Konrad RJ: Fenofibrate treatment increases human serum proprotein convertase subtilisin kexin type 9 levels. J Lipid Res 2010, 51:345-351.

13. Browning JD, Horton JD: Fasting reduces plasma proprotein convertase, subtilisin/kexin type 9 and cholesterol biosynthesis in humans. J Lipid Res 2010, 51:3359-3363.

14. Cui Q, Ju X, Yang T, Zhang M, Tang W, Chen Q, Hu Y, Haas JV, Troutt JS, Pickard RT, Darling R, Konrad RJ, Zhou H, Cao G: Serum PCSK9 is associated with multiple metabolic factors in a large Han Chinese population. Atherosclerosis 2010, 213:632-636.

15. Miyazawa H, Honda T, Miyauchi S, Domon H, Okui T, Nakajima T, Tabeta K, Yamazaki K: Increased serum PCSK9 concentrations are associated with periodontal infection but do not correlate with LDL cholesterol concentration. Clin Chim Acta 2012, 413:154-159.

16. Feingold KR, Moser AH, Shigenaga JK, Patzek SM, Grunfeld C: Inflammation stimulates the expression of PCSK9. Biochem Biophys Res Commun 2008, 374:341-344.

17. Le Bras M, Roquilly A, Deckert V, Langhi C, Feuillet F, Sébille V, Mahé PJ, Bach K, Masson D, Lagrost L, Costet P. Asehnoune K, Cariou B: Plasma PCSK9 is a late biomarker of severity in patients with severe trauma injury. J Clin Endocrinol Metab 2013, 98:E732-E736.

18. Chen SN, Ballantyne CM, Gotto AM Jr, Tan Y, Willerson JT, Marian AJ: A common PCSK9 haplotype, encompassing the E670G coding single nucleotide polymorphism, is a novel genetic marker for plasma low-density lipoprotein cholesterol levels and severity of coronary atherosclerosis. J Am Coll Cardiol 2005, 45:1611-1619.

19. Duan HF, Wang H, Yi J, Liu HJ, Zhang QW, Li LB, Zhang T, Lu Y, Wu CT, Wang LS: Adenoviral gene transfer of sphingosine kinase 1 protects heart against ischemia/reperfusion-induced injury and attenuates its postischemic failure. Hum Gene Ther 2007, 18:1119-1128.

20. Guo YL, Liu J, Xu RX, Zhu CG, Wu NQ, Jiang LX, Li Jj: Short-Term Impact of Low-Dose Atorvastatin on Serum Proprotein Convertase Subtilisin/Kexin Type 9. Clin Drug Investig 2013, 33:877-883.

21. Jeong HJ, Lee HS, Kim KS, Kim YK, Yoon D, Park SW: Sterol dependent regulation of proprotein convertase subtilisin/kexin type 9 by sterol regulatory element binding-protein 2. J Lipid Res 2008, 49:399-409.

22. Li H, Dong B, Park SW, Lee HS, Chen W, Liu J: Hepatocyte nuclear factor 1 alpha plays a critical role in PCSK9 gene transcription and regulation by the natural hypocholesterolemic compound berberine. J Biol Chem 2009, 284:28885-28895.

23. Li JJ: Inflammation in coronary artery diseases. Chin Med J (Engl) 2011, 124:3568-3575.

24. Klingenberg R, Luscher TF: Inflammation in coronary artery disease and acute myocardial infarction - is the stage set for novel therapies? Curr Pharm Des 2012, 18:4358-4369.

25. Blancke F, Claeys MJ, Jorens P, Vermeiren G, Bosmans J, Wuyts FL, Vrints CJ: Systemic inflammation and reperfusion injury in patients with acute myocardial infarction. Mediat Inflamm 2005, 2005:385-389.

26. Yavuz MT, Yavuz O, Yazici M, Guler S, Ozhan H, Albayrak S, Coskun A: Interaction between Chlamydia pneumoniae seropositivity, inflammation and risk factors for atherosclerosis inpatients with severe coronary stenosis. Scand J Clin Lab Invest 2006, 66:523-534.

27. Li JJ, Wang HR, Huang CX, Xue JL, Li GS: Enhanced response of blood monocytes to C-reactive protein in patients with unstable angina. Clin Chim Acta 2004, 352:127-133.

28. Li JJ, Fang CH, Cheng MZ, Chen X, Lee SW: Activation of nuclear factor-kB and correlation with elevated plasma C-reactive protein in patients with unstable angina. Heart Lung Circ 2004, 13:173-178.

29. Altun B, Turkon H, Tasolar H, Beggı H, Altun M, Temız A, Gazı E, Barutcu A, Bekler A, Colkesen Y: The relationship between high-sensitive troponin T, neutrophil lymphocyte ratio and SYNTAX Score. Scand I Clin Lab Invest 2014, 74:108-115.

30. Baldus S, Heeschen C, Meinertz T, Zeiher AM, Eiserich JP, Münzel T, Simoons ML, Hamm CW, CAPTURE Investigators: Myeloperoxidase serum levels predict risk in patients with acute coronary syndromes. Circulation 2003, 108:1440-1445.

31. Zhu XY, Zhang ZL, Li P, Liang WY, Feng XR, Liu ML: Shenyuan, an extract of American Ginseng and Corydalis Tuber formula, attenuates 
cardiomyocyte apoptosis via inhibition of endoplasmic reticulum stress and oxidative stress in a porcine model of acute myocardial infarction. J Ethnopharmacol 2013, 150:672-681.

32. Rousselet E, Marcinkiewicz J, Kriz J, Zhou A, Hatten ME, Prat A, Seidah NG: PCSK9 reduces the protein levels of the LDL receptor in mouse brain during development and after ischemic stroke. J Lipid Res 2011, 52:1383-1391.

33. Wu CY, Tang ZH, Jiang L, Li XF, Jiang ZS, Liu LS: PCSK9 siRNA inhibits HUVEC apoptosis induced by ox-LDL via BCl/Bax-caspase9-caspase3 pathway. Mol Cell Biochem 2012, 359:347-358.

34. Khovidhunkit W, Kim MS, Memon RA, Shigenaga JK, Moser AH, Feingold KR, Grunfeld C: Effects of infection and inflammation on lipid and lipoprotein metabolism: mechanisms and consequences to the host. J Lipid Res 2004, 45:1169-1196.

35. Ferri N, Tibolla G, Pirillo A, Cipollone F, Mezzetti A, Pacia S, Corsini A, Catapano AL: Proprotein convertase subtilisin kexin type 9 (PCSK9) secreted by cultured smooth muscle cells reduces macrophages LDLR levels. Atherosclerosis 2012, 220:381-386.

36. Berti V, Sciagrà R, Acampa W, Ricci F, Cerisano G, Gallicchio R, Vigorito C, Pupi A, Cuocolo A: Relationship between infarct size and severity measured by gated SPECT and long-term left ventricular remodelling after acute myocardial infarction. Eur I Nucl Med Mol Imaging 2011, 38:1124-1131.

37. Ballantyne CM, Pitt B, Loscalzo J, Cain VA, Raichlen JS: Alteration of relation of atherogenic lipoprotein cholesterol to apolipoprotein $B$ by intensive statin therapy in patients with acute coronary syndrome (from the Limiting UNdertreatment of lipids in ACS With Rosuvastatin [LUNAR] Trial). Am J Cardiol 2013, 111:506-509.

38. Ferrières J, Cambou JP, Guéret P, Boutalbi Y, Lablanche JM, Hanania G, Genès N, Cantet C, Danchin N: Effect of early initiation of statins on survival in patients with acute myocardial infarction (the USIC 2000 Registry). Am J Cardiol 2005, 95:486-489.

doi:10.1186/1471-2261-14-192

Cite this article as: Zhang et al.: Proprotein convertase subtilisin/kexin type 9 expression is transiently up-regulated in the acute period of myocardial infarction in rat. BMC Cardiovascular Disorders 2014 14:192.

\section{Submit your next manuscript to BioMed Central and take full advantage of:}

- Convenient online submission

- Thorough peer review

- No space constraints or color figure charges

- Immediate publication on acceptance

- Inclusion in PubMed, CAS, Scopus and Google Scholar

- Research which is freely available for redistribution 